\title{
AIDS in 2003:
}

\section{Moving Forward, Falling Back}

\author{
Patricia M. Spittal, PhD \\ Martin T. Schechter, OBC, MD, PhD, FRCPC
}

$\mathrm{T}$ The history of the AIDS epidemic has been shaped by an ongoing dialectic between moving forward in some areas, while at the same time falling back in others. ${ }^{1}$ Regrettably today, we must report that the world continues to face the same tension between progress and failure in its enduring efforts to control and overcome this pandemic.

A vivid microcosm of this appears in the pages of this issue of the Canadian Journal of Public Health. Herein, we find two articles, one chronicling one of the major successes achieved thus far, the other demonstrating the ongoing challenges we face. Dena Schanzer (pages 135-39) presents a thorough account of trends in AIDS mortality in Canada over the 11 years from 1987 through 1998. Her analysis, as highlighted in Figure 1 of her article, demonstrates the breathtaking success that was achieved in reducing HIV mortality in the mid 1990s. HIV/AIDS deaths declined 65\% between 1995 and 1997. These declines in HIV/AIDS mortality (Figure 4) and in person-years of life lost to HIV infection (PYLL; Figure 5) far outstripped any gains we made with respect to Canada's other major killers. These accomplishments are clearly attributable to the advent of protease inhibitors and highly aggressive anti-retroviral therapy (HAART) regimens that became widely available in Canada during this critical period. Medical science has produced relatively few agents that are effective against viruses, let alone retroviruses. In light of this, the development and dissemination of highly effective anti-HIV drug combinations, within 11 years of the discovery of this previously unknown and formidable retrovirus, represents one of the most stunning successes in the history of the pandemic.

Yet in the pages of this same issue, we see evidence of continuing deficiencies. The survey of Ontario physicians reported by Guenter and colleagues (pages 93-97) demonstrates that as recently as July 2000, significant gaps in physician knowledge about prenatal HIV testing still persist. While this might not have been such a concern in bygone days when we had little to offer in the way of perinatal prevention, this is now far from the case. Variations of the same drug regimens described above are now able to virtually eradicate perinatal transmission, with rates as low as $1 \% .^{2}$ It is therefore disconcerting to see that as many as $47 \%$ of surveyed physicians were unaware of the province's prenatal testing poli$c y$, and that a significant proportion did not routinely offer HIV counselling and testing to pregnant women.

Throughout the world in 2003, we are continuing to see this juxtaposition of moving forward in some areas, while at the same time failing in others. Though the combination therapies described earlier have represented a therapeutic miracle, their benefits have reached only a tiny minority of people with HIV around the world. While some progress has been made in making these treatments available in middle income countries, Brazil being a notable example, they remain far out of reach for most developing countries where 95\% of people with HIV infection live. For this reason, we can expect more than 3 million

Department of Health Care and Epidemiology, Faculty of Medicine, UBC and the BC Centre for Excellence in HIV/AIDS, St. Paul's Hospital, Vancouver, BC

Correspondence: Dr. Martin Schechter, Dept. of Health Care and Epidemiology, Faculty of Medicine, University of British Columbia, 5804 Fairview Avenue, Vancouver, BC V6T 1Z3 Tel: 604-822-3910, Fax: 604-822-4994, E-mail: martin.schechter@ubc.ca

Acknowledgments: Dr. Schechter is supported by a tier I Canada Research Chair in HIV/AIDS and Urban Population Health

people including 600,000 children to die of their HIV infection in 2003, almost all of them in the developing world. ${ }^{3}$

On the prevention front, we see the same combination of progress and failure. Prevalence rates are continuing to decline in countries such as Uganda, Zambia, Thailand and Cambodia. More recently, in Addis Ababa, Ethiopia, infection levels among women aged 15-24 attending antenatal clinics dropped from $24.2 \%$ in 1995 to $15.1 \%$ in 2001.4 Almost always, these gains occur when governments overcome denial and are willing to acknowledge the HIV/AIDS problem openly and exercise the political will to confront it. However, while this progress was being made, ominous spread of HIV was occurring elsewhere in the world. There has been rapid spread of HIV, primarily via injection drug use, throughout the newly independent states of the former Soviet Union. ${ }^{5}$ Moreover, the past few years have witnessed dramatic spread of HIV in India, China, and Indonesia. It is estimated that 4 million people in India are now HIV infected, with another 1 million infected in China. ${ }^{3}$ In Jakarta, the prevalence of HIV among injection drug users rose from very low levels in 1998 to as high as 50\% by 2001 . While the epidemic remains localized within these countries at the present time, ${ }^{3}$ their collective total population exceeds 2.5 billion people, a huge potential target for HIV. Even if the prevalence in these countries only rose to $5 \%$, this would represent 125 million people, more than triple the number of people who have been infected to date.

Regrettably, there is only bad news for the family members and communities left behind by the pandemic. A total of about 15 million children will have been orphaned by AIDS by the end of 2003. A study in Zambia found that $65 \%$ of households dissolved following the death of the mother. ${ }^{3}$ Household income has been found to fall by $80 \%$ or more in the majority of households where the father has died. ${ }^{3}$ Strategies to deal with the excessive medical and burial costs often include selling off remaining assets such as land and animals, and removing children, particularly girls, from school so that they can generate income. A grim milestone may be achieved this year or next when the number of prevalent and incident cases of HIV in women could exceed those in men. This 
has already occurred in Africa. ${ }^{3}$ The impact will be enormous, especially given that, in rural areas of sub-Saharan Africa and Asia, women contribute more than $50 \%$ to food production and are the cornerstone of agrarian societies. Inexorably, the social, economic and demographic shocks and aftershocks of HIV will be felt around the world for decade upon decade to come.

A glimmer of hope appeared in June 2001 when the United Nations General Assembly held a Special Session about the crisis and published its Declaration of Commitment on HIV/AIDS. ${ }^{6}$ This document contains the words "We, heads of State and Government and representatives of States and Governments...solemnly declare our commitment to address the HIV/AIDS crisis by taking action as follows." 6 The document went on to list
67 actions that each country vowed to undertake, including the establishment of an annual global fund of $\$ 7$ to $\$ 10$ billion US to combat the pandemic. For the first time, wealthy countries had officially adopted a course of action and committed resources on a global scale. It was regrettable that one year later, Canada's Stephen Lewis, the United Nations Special Envoy for HIV/AIDS in Africa, worriedly reported that only $\$ 2.1$ billion US had been pledged to the fund, and only $\$ 300$ million US were actually received. ${ }^{7}$ Commitment means moving forward; lip service means falling back.

\section{REFERENCES/REFERENCES}

1. Strathdee SA, Schechter MT. HIV/AIDS -Year in Review: One step forward, two steps back. [E] Can Med Assoc J 1997;157:1699-700.
2. Mofenson LM. U.S. Public Health Service Task Force recommendations for use of antiretroviral drugs in pregnant HIV-1-infected women for maternal health and interventions to reduce perinatal HIV-1 transmission in the United States. MMWR Recomm Rep 2002;51(RR-18):1-38.

3. Joint United Nations Programme on HIV/AIDS (UNAIDS). AIDS Epidemic Update 2002. World Health Organization, Geneva, December 2002. (http://www.unaids.org)

4. Tsegaye A, Rinke De Wit TF, Mekonnen Y, et al. Decline in prevalence of HIV-1 infection and syphilis among young women attending antenatal care clinics in Addis Ababa, Ethiopia: Results from sentinel surveillance, 1995-2001. JAIDS Journal of Acquired Immune Deficiency Syndromes 2002;30:359-62.

5. Kerr C. Heading for disaster: HIV prevalence soars in eastern Europe. The Lancet Infectious Diseases 2002;2:204.

6. United Nations. Declaration of Commitment on HIV/AIDS. Special Session, New York, June 2527, 2001. http://www.unaids.org/UNGASS/ index.html

7. Lewis $S$. From a speech at a satellite symposium "Financing the AIDS Crisis" at the XIV International AIDS Conference, Barcelona, July 7-12, 2002.

\title{
Le sida en 2003 : quelques pas en avant, quelques pas en arrière
}

\author{
Patricia M. Spittal, PhD \\ Martin T. Schechter, OBC, MD, PhD, FRCPC
}

$\mathrm{L}$ 'histoire de l'épidémie de sida a été marquée par la dialectique entre les avancées dans certains domaines et les reculs dans d'autres. ${ }^{1}$ Malheureusement, aujourd'hui tout indique que le monde vit encore la même tension entre progrès et échecs dans ses efforts persistants pour maîtriser et vaincre cette pandémie.

On retrouve la même dialectique, en microcosme, dans les pages de ce numéro de la Revue canadienne de santé publique. De deux articles sur le sujet, l'un relate l'une de nos grandes réussites jusqu'à maintenant, et l'autre explique les défis qu'il nous reste encore à relever. Dena Schanzer (pages 135 à 139) présente un compte rendu exhaustif des tendances de la mortalité due au sida au Canada sur une période de 11 ans (1987-1998). Son analyse, comme on le voit à la figure 1 de l'article, met en évidence la réussite époustouflante qu'a été la réduction de la mortalité due au VIH au milieu des années 1990. Les décès liés au $\mathrm{VIH} /$ sida ont diminué de $65 \%$ entre 1995 et 1997 . Ces baisses de la mortalité due au $\mathrm{VIH} /$ sida (figure 4) et des années de vie perdues en raison des infections à VIH (PYLL; figure 5) ont surpassé de loin les progrès réalisés à l'égard des autres grandes maladies mortelles au Canada. Ces exploits sont clairement liés à l'avènement des inhibiteurs de pro-

Département des soins de santé et d'épidémiologie de la faculté de médecine de l'Université de la Colombie-Britannique et BC Centre for Excellence in HIV/AIDS, hôpital St. Paul's, Vancouver (C.B.)

Correspondance : Dr Martin Schechter, Dept. of Health Care and Epidemiology, Faculty of Medicine, University of British Columbia, 5804 Fairview Avenue, Vancouver (Colombie-Britannique) V6T 1Z3, tél. : 604-822-3910, téléc. : 604-822-4994, courriel : martin.schechter@ubc.ca

Remerciements : Le Dr Schechter est titulaire d'une chaire de recherche du Canada de niveau 1 sur le $\mathrm{VIH} /$ sida et la santé des populations urbaines.

téase et des traitements antirétroviraux hautement actifs, qui se sont diffusés à grande échelle au Canada durant cette période critique. La science médicale a découvert relativement peu d'agents efficaces contre les virus, a fortiori contre les rétrovirus. Par conséquent, la mise au point et la diffusion d'associations de médicaments anti-VIH très efficaces, à peine 11 ans après la découverte d'un rétrovirus formidable et inconnu jusque là, comptent parmi les réussites les plus marquantes de l'histoire de cette pandémie.

Ce même numéro renferme cependant un constat de lacunes. L'enquête auprès des médecins ontariens dont Guenter et ses collègues (pages 93 à 97) font le compte rendu montre qu'aussi récemment qu'en juillet 2000, il subsistait des écarts importants dans la connaissance du dépistage anténatal du VIH par les médecins. Ce n'était pas vraiment préoccupant à l'époque où il n'existait presque rien en matière de prévention périnatale, mais c'est loin d'être le cas maintenant. Avec des variantes des régimes posologiques mentionnés plus haut, on peut désormais pratiquement éradiquer la transmission périnatale (les cas réfractaires ne seraient plus que $1 \%){ }^{2}$ Il est donc déconcertant de voir qu'un bon $47 \%$ des médecins interrogés n'étaient pas au courant de la politique provinciale de 
dépistage anténatal, et qu'une proportion importante n'aiguillaient pas systématiquement les femmes enceintes vers les services de counselling et de dépistage du VIH.

À l'échelle mondiale en 2003, on constate aussi des progrès à certains égards et des reculs ailleurs. Les polythérapies mentionnées plus haut ont eu des effets miraculeux, mais seule une infime minorité de personnes atteintes du VIH en ont profité autour du monde. On a réussi à offrir ces traitements dans des pays à revenu intermédiaire, tout particulièrement au Brésil, mais ils sont toujours hors de la portée de la plupart des pays en développement, où se concentrent $95 \%$ des personnes infectées par le VIH. C'est pourquoi on prévoit que plus de trois millions de personnes, dont 600000 enfants, mourront de leur infection à VIH en 2003, presque tous dans le monde en développement. ${ }^{3}$

Sur le front de la prévention, on voit la même combinaison de progrès et d'échecs. Les taux de prévalence continuent à baisser dans des pays comme l'Ouganda, la Zambie, la Thaïlande et le Cambodge. À Addis-Ababa (Éthiopie), les niveaux d'infection chez les femmes de 15 à 24 ans qui fréquentent les cliniques de soins prénataux sont passés de 24,2 \% en 1995 à $15,1 \%$ en $2001 .{ }^{4}$ Presque toujours, ces gains surviennent lorsque les gouvernements cessent de nier la réalité, qu'ils sont disposés à reconnaître ouvertement le problème du VIH/sida et qu'ils ont la volonté politique de s'y attaquer. Tandis que l'on progressait sur ce front cependant, le VIH a continué de faire des ravages ailleurs dans le monde. Partout dans les États nouvellement indépendants de l'an- cienne Union soviétique, sa propagation a été rapide et s'est faite principalement par l'utilisation de drogues injectables. ${ }^{5}$ Le VIH s'est aussi propagé de façon fulgurante en Inde, en Chine et en Indonésie ces dernières années. Selon les estimations, quatre millions de personnes auraient maintenant contracté l'infection en Inde, et un million en Chine. ${ }^{3}$ À Jakarta, la prévalence du VIH chez les utilisateurs de drogues injectables, qui était très faible en 1998, avait grimpé à $50 \%$ en 2001. Dans ces pays, l'épidémie est encore circonscrite pour l'instant, ${ }^{3}$ mais les 2,5 milliards d'habitants qu'ils comptent collectivement représentent une énorme cible potentielle pour le VIH. Même si la prévalence dans ces pays n'atteignait que $5 \%$, cela représenterait 125 millions de personnes, plus que trois fois le nombre de personnes infectées jusqu'à maintenant.

Malheureusement, les nouvelles sont uniformément mauvaises pour les familles et les collectivités que la pandémie aura laissés dans son sillage. D'ici la fin de 2003, le sida aura rendu une quinzaine de millions d'enfants orphelins. Selon une étude réalisée en Zambie, $65 \%$ des ménages se dissolvent après le décès de la mère. ${ }^{3}$ Et le revenu baisse de $80 \%$ ou plus dans la majorité des ménages où le père est décédé. $^{3}$ Pour payer les coûts exhorbitants des soins médicaux et de l'inhumation, on doit se résigner à vendre ses biens restants (sa terre, son bétail) et à retirer ses enfants de l'école, particulièrement les filles, pour leur permettre de gagner un revenu. Un bien triste jalon pourrait être atteint cette année ou l'an prochain lorsque le nombre de cas de prévalence et d'incidence du VIH chez les femmes dépassera celui des hommes. On y est déjà en Afrique. ${ }^{3}$ Les répercussions seront énormes, surtout que dans les zones rurales de l'Afrique subsaharienne et de l'Asie, les femmes contribuent plus de la moitié de la production vivrière et sont la pierre angulaire des sociétés agraires. Inexorablement, les chocs et les ondes de choc du VIH se feront sentir dans le monde entier, sur les plans social, économique et démographique, pendant plusieurs décennies.

Une lueur d'espoir est apparue en juin 2001 lorsque l'Assemblée générale des Nations Unies a tenu une session extraordinaire sur la crise et publié sa Déclaration d'engagement sur le VIH/sida. ${ }^{6}$ On y affirme que " Nous, chefs d'État et de gouvernement et représentants d'État et de gouvernement... déclarons solennellement que nous nous engageons à rechercher des solutions à la crise du VIH/sida en adoptant les mesures suivantes. " ${ }^{6}$ Le document énumère ensuite 67 mesures que chaque pays s'engage à entreprendre, dont la création d'un fonds mondial de 7 à 10 milliards \$US par année pour lutter contre la pandémie. Pour la première fois, les pays riches ont officiellement adopté un plan d'action et engagé des ressources à l'échelle mondiale. Il est regrettable qu'un an plus tard, le Canadien Stephen Lewis, envoyé spécial des Nations Unies pour le VIH/sida en Afrique, rapportait avec inquiétude que les pays n'ont promis que des versements de 2,1 milliards \$US dans le fonds et n'y ont encore versé que 300 millions de dollars. ${ }^{7}$ L'engagement est un pas en avant; les simples paroles nous font revenir en arrière. 